\title{
Quality of Service Study in Synchronized Time-Triggered Aerial Networks
}

\author{
Nahman Tariq \\ School of Aerospace,Transport \\ and Manufacturing (SATM) \\ Cranfield University \\ nahman.tariq@cranfield.ac.uk
}

\author{
Ivan Petrunin, \\ School of Aerospace,Transport \\ and Manufacturing (SATM) \\ Cranfield University \\ i.petrunin@cranfield.ac.uk \\ Wolfram Zischka \\ TTTech \\ Vienna, Austria \\ wolfram.zischka@tttech.com
}

\author{
Saba Al-Rubaye \\ School of Aerospace, Transport \\ and Manufacturing (SATM) \\ Cranfield University \\ s.alrubaye@cranfield.ac.uk
}

\author{
Antonios Tsourdos \\ School of Aerospace, Transport \\ and Manufacturing (SATM) \\ Cranfield University \\ a.tsourdos@cranfield.ac.uk
}

\begin{abstract}
Unmanned aerial vehicles (UAVs) have shown tremendous potential in both military and civilian applications, for example, in delivery services, search and rescue, and surveillance. Currently, UAVs can perform several tasks while in flight. UAVs have real-time systems with limited capability for time-triggered synchronization, which impose synchronization limitations for their platforms. Additionally, there is no timetriggered synchronization between the UAVs and the ground station. One of the requirements for a reliable communication links between a UAV and a ground station is the need for synchronization using Global Navigation Satellite System (GNSS) in a time-triggered network. However, the information exchange between UAV and ground station lacks the time-triggered abilities. In this context, GNSS time can be used as an external source to allow UAV performing tasks connected to the ground station with time-stamped messages. In this paper, we investigate the capability of GNSS as an external time source for the timetriggered network to achieve high quality of service (QoS) of a communication link between the ground station and UAV. Simulations analysis was conducted to evaluate the overall system performance, which gives a better understanding of performance of a time-triggered wired and wireless network.
\end{abstract}

Keywords- Time-Triggered, GNSS, UAVs, Network, System of Systems, Quality of Service, Virtual Links

\section{INTRODUCTION}

An unmanned aerial vehicles (UAVs), also referred to as drones, are widely used in multiple applications and the usage of UAV's has increased significantly in the past decade. Rreliable connection between the ground station and UAV is one of a key challenges that are yet to be addressed. One of the potential solutions for improved communication flexibility, reliability, and robustness in wireless aerial networks based on UAVs is to use an external GNSS [1]. A Time-Triggered Ethernet (TTE), is a highly reliable network, which supports time-triggered messages to be delivered in a given global time base. Time synchronization is an important part of distributed systems. Such systems have different requirements to the accuracy of time synchronization. Time-triggered architecture can provide synchronization for multiple computing nodes to perform different tasks and exchange messages, in order to cooperate with each other [1]. An example of such architecture is the precision time protocol (PTP). This protocol is applicable to distributed systems, consisting of one or more nodes communicating over the network [2].

Several other standard solutions available as a reliable interconnect are including Time-Triggered Protocol(TTP) [3] and Aeronautical Radio, Incorporated (ARINC 664) [4]. With growing need in bandwidth and demand for integrating different levels of criticality in a single physical network, these solutions are being used in a broad variety of systems. The combination of networks (e.g. UAVs with TT networks) forms a system of systems (SoS), which can provide the capability of TTEthernet (TTE) deployment and incorporates best standards for supporting a fault tolerant and a reliable communications network. TTE can be used in communication platforms and it integrates multiple systems capable of hosting applications with different time and safety-criticality requirements.

The transmission of TT traffic in TTE adheres to a strict scheduling scheme. However, when TT traffic sent from a synchronisation master (SM) misses its pre-allocated timetriggered window, it has to wait for its next scheduling round. Time-Triggered Ethernet (TTE) can be applied as the communication network for distributed systems, since it possesses time integrity guarantee mechanism, matches the processing isolation of partitions, and supports mixed criticality traffic, including Time-Triggered (TT) traffic, Rate-Constrained (RC) traffic and Best-Effort (BE) traffic [5]. As discussed above the importance of TT message in a distributed system is in knowing how the messages are communicated in the network. We know what type of messages are present in the network, but the important part is how these messages are communicated. A TT network consists of end systems (ES) and switches (SW). These ES and SW work both as a synchronisation master (SM) and client master (CM). SM and $\mathrm{CM}$ support efficient communication that takes place in the network; SM also makes sure that all the clocks in ES are synchronised and all the CMs are synchronised with the correct time too. In autonomous mode network is using its own autonomous time as the main clock source for synchronisation. 


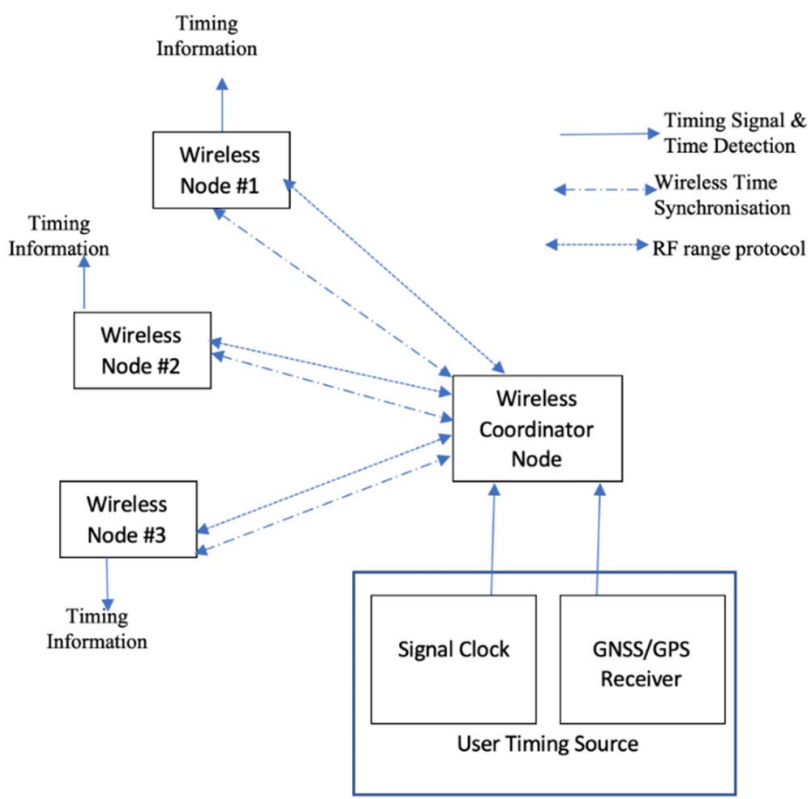

Fig. 1. Wireless network clock synchronisation.

The wireless system has a single time source responsible for transmission of a timing message from coordinator to the connected nodes, as shown in Figure 1. Wireless nodes identify the timing message when received and synchronize the wireless node to time on the timing message.

In this paper, we explore different methods for GNSS to be used as an external time source with synchronized timetriggered traffic, which allows UAV to perform tasks in connection to the ground station with predefined time. The main contributions of this paper is in analysis of the system latency and recommendations as for improvement of the overall performance and quality of service (QoS), e.g. by connecting multiple networks to support time-triggered traffic with and without GNSS support as the main time source.

The rest of the paper is organized as follows: Section I describes time-triggered aerial networks. In Section II, the system design overview is presented, and the simulation analysis are demonstrated in In Section III. In Section IV simulations are conducted and results are presented. Finally, concluding remarks and possible future work are presented.

\section{Time-Triggered AERIAL NETWORKS}

In the TT network time synchronization only needs to be configured if time-triggered traffic is to be transmitted in the network. A TTE network according to the SAE AS6802 (TimeTriggered Ethernet) standard can have multiple synchronization domains and multiple synchronization priorities [5]. The use of multiple synchronization domains allows for configuring independent time-triggered networks within one aerial network domain and create individual schedules.

The Time-Triggered synchronization messages maintain the clock synchronization [6] during the operational phase of the TT Ethernet system. A synchronization block, i.e., a tight sequence of one to five synchronization messages-corresponding to the one to five time-keeping controllers-is sent periodically. The time-triggered protocol uses a pre-defined clock which allows the entire network to synchronise with the autonomous time. The time-triggered clock-synchronization protocol maintains the synchronized global time [7]. This protocol operates after initial synchronization is reached, that is, once the local clocks of the components are already synchronized with known bounds.

The operation of any time-triggered system depends on the availability of a global time base. Any loss of the time base is tantamount to system failure. In safety critical applications the establishment of a fault-tolerant time-base of a priori known precision is thus a definite requirement [8].

Several proposals have been made to overcome the limitations of 802.11 MAC in regard to deterministic data transmission. One of the most commonly found MAC protocols in wireless networks is carrier sense multiple access with collision avoidance (CSMA/CA). It is used as part of the widely known standards IEEE 802.11 for LAN and IEEE 802.15.4 for wireless personal area networks (WPANs), with the benefit that it is not too complex to implement, and in lightly loaded settings it can achieve good throughput. However, CSMA does not provide a predictable channel access delay since access to the medium is random and packets can collide[9]. Some other techniques are focused on improving the admission control and scheduling algorithms of HCF Coordinated Chanel Access (HCCA) (i.e. [10] and [11]). One of the most promising solutions is IsoMAC a TDMA approach based on 802.11e MAC (without HCCA), that provides a way to satisfy soft real-time flows [12].

It is important to take propagation delay into account when designing a time synchronization protocol for an aerial network system such as UAV. The term propagation delay to refer to the duration a signal travels between the antennas of two communication partners. First, we quantify the propagation delay in wireless embedded systems and compare it against other errors present when synchronizing time in multi-hop networks [13] [14].

An important aspect when dealing with flying object is the data link QoS. Therefore, UAV should consider reliable data transmissions to and from the ground station and thus a communication path should be available at any moment with real time synchronization. The TTEthernet networks sometimes become a challenge with a requirement of coordinated wireless communication on remote locations. TTEthernet is designed to tolerate either the failure of a bridge or an end station, but not multiple failures at the same time. If end stations have inconsistent omission failures, then TTEthernet can tolerate two simultaneous component failures. Hence, multiple access points need to be synchronized to each other. For reliability reasons, fault-tolerance and robustness mechanisms different studies has been done in this area e.g., the wireless communication needs to tolerate the temporary or permanent loss of wireless components, like access points [7]. After considering the abovementioned techniques and challenges, we have tried to use a combination of these mechanisms which include an GNSS as external resources to support wireless data link of UAV and to 
provide better understating of the QoS in the overall aerial network.

\section{SYSTEM DESIGN OVERVIEW}

To overcome the problem of system synchronisation of multiple TTE network methods, it is necessary for each UAV to have capabilities to communicate with the ground station. The proposed system design allows wireless end system (WES) to be connected to the main TTE network. As shown in Figure 2, the end stations (ESs) are connected to fully redundant networks. A GNSS receiver is used to enable connectivity to (ES1). TTEthernet provides several services to improve its realtime and fault tolerance performance. In particular, TTE implements synchronization protocols that establish and maintain synchronized time in the network in a fault-tolerant manner [6]. The system design overview shows the communication between ES, switches, and physical links (Ethernet). Each ES is connected with a switch via a physical link, connectivity between GNSS receiver and ES1 is through USB, which introduces USB stack latency.

TTEthernet implements synchronization protocols that establish and maintain synchronized time in the network in a fault-tolerant manner. Typically, the SM functionality is implemented in the end stations (ES) while some of the bridges operate as CMs. In Figure 2, the ESs are configured as SMs while bridges $\mathrm{A} 1$ and $\mathrm{B} 1$ are configured as $\mathrm{CMs}$; bridge $\mathrm{B} 2$ operates as an SC. TTE differentiates between SMs, CMs and synchronisation clients (SCs). Typically, the SM functionality is implemented in the ESs while some of the bridges operate as CMs.
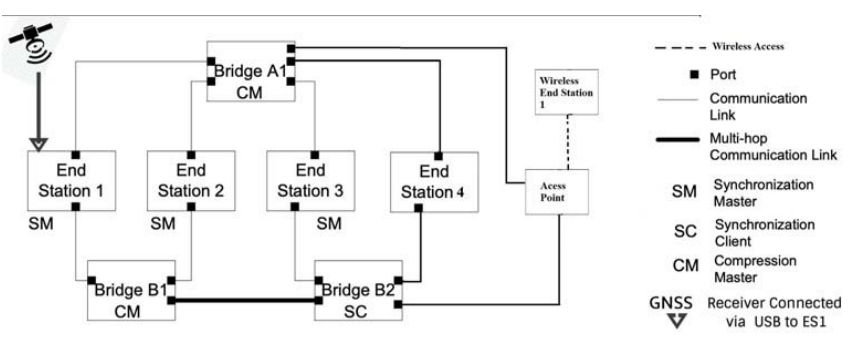

Fig. 2. Overview of TTE simulation network.

The end stations are connected to fully redundant networks. In this case, network A consists of a single bridge, while network B consists of two bridges connected to each other with a multihop link. The synchronization protocols exchange "protocol control frames" (PCFs) between end stations and bridges in order to establish a synchronized time base in the system. Similarly, the system design is based on one difference that is introduction of time from an external source in this case a GNSS which connected to ES1 via USB. The third scenario is a GNSS device connected to ES1 and a WES connected via access point with the TTE network. Network A consists of a single bridge, while network B consists of two bridges connected to each other with a multi-hop link. The synchronization protocols exchange "protocol control frames" (PCFs) between end stations and bridges in order to establish a synchronized time base in the system. The PCFs follow the standard Ethernet frame format and as a most basic rule, all end stations always send their PCFs to all their connected networks.

During system operation, each sender monitors the network time and dispatches a time-triggered frame whenever the current network time matches an entry in the communication schedule. The network time is thus of critical importance for timetriggered communication. Protocols that establish and maintain network time must be in place. such protocols are generally called "synchronization protocols". The third configuration in the network which is our key focus is the introduction of wireless end station (WES) in the TTE network. The proposed system design represents this by incorporating an access point which allows the WES to be connected to the TTE network.

The reason for selecting TTE as the best suitable candidate to have aerial SoS network is in support of multiple traffic types. For example, the aerospace version of TTEthernet supports both standard best-effort Ethernet communication, which provides no guarantees on jitter or latency and the ARINC 664-p7 communication paradigm (also called rate-constrained traffic) for which one can compute bounds on worst-case latency and jitter. But we are interested in latency of the network, showing how it effects QoS in the SoS.

\section{SimUlATION}

Simulations are carried out using the TTTech development system to, achieve and compare the performance of three scenarios, which are identified as one being wireless network, second, being GNSS as external clock synchronization in the time-triggered network and lastly a TTE network its own synchronization clock. This correction is then applied, and correction is propagated to each device through the timetriggered network, and the global clock is updated. The jitter of a VL cannot exceed 500 us in a source ES. In the system analysis, we identify parameters (e.g., latency) that can affect the data communication link in the network. These parameters help us to compare the performance of QoS in a system of systems application with internal (through wireless link) and external (by GNSS) synchronization. The simulation using the TTTech development system is implemented to perform the system analysis. Moreover, the simulations were conducted with and without GNSS synchronization to allow us to identify a system of systems (SoS) performance. TTTech has provided us with the development system which allows us to perform TT based experiments and allows us to simulate various network traffic related experiments. More details about the TTTech development system can be read here [15].

The simulation were carried out in three different steps, internal clock synchronisation, GNSS external clock synchronisation and GNSS external clock with a WES. The simulation design works uses an internal clock and also an external GNSS clock for synchronisation. In this simulation we define multiple parameters for the network to perform TT related tasks to identify QoS in the network. We use 50 second simulation time period which provides us with a detailed enough result for understanding QoS latency in the SoS network. As we can identify header bytes of the TT message for all the simulation, we use 16 and 64 bytes as the header size for the TT messages in the network. As shown in the system design we 
proposed three scenarios with the set parameters mentioned above. one key point which is important to understand is the message header bytes pay an important role when the TT messages are propagated in the network the bigger the header byte latency increases in the network effecting the overall performance of the network. The most suitable header size was used so latency is not affected in a dramatic way this was the reason for using 16 and 64 bytes as the header message size. The scenario presented in Figure 2 is of a wired TTE network this allows for an internal clock synchronisation. For first simulation we use 16 and 64 bytes as message header for TT messages in a 50 second simulation.

\section{RESUlts AND ANALYSIS}

In this section we describe and analyse the results of the simulation, which is discussed in the previous section. The focus here is on how the QoS is measured in the TTE network, which supports multiple networks as well as having an external clock synchronisation via GNSS and having a WES connected to the main TTE network using an access point. The results shown in Figures below utilise both 16 and 64 bytes length of message header.

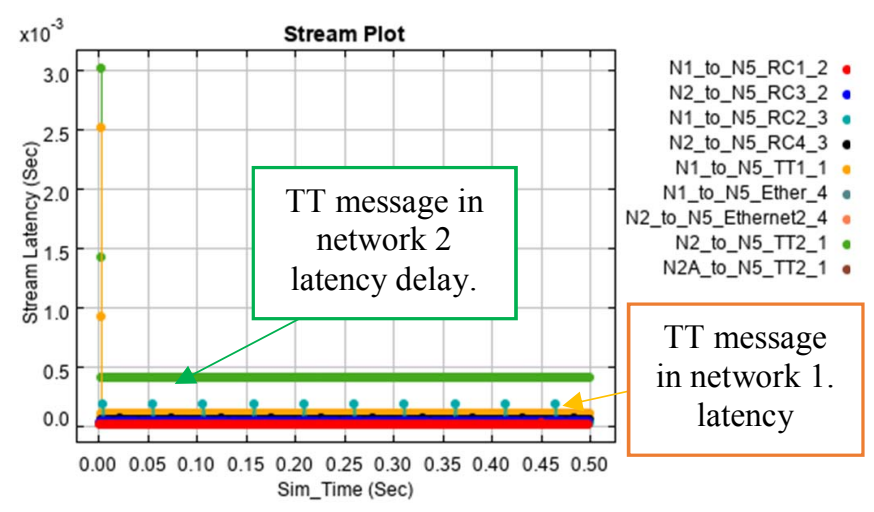

Fig. 3. Proposed scenario with multiple TTE networks in a wired connection.

Figure 3 presents a stream plot of a wired TT network. The proposed simulation as discussed before is of multiple network. In the above Figure we highlight the latency in two networks one being orange which represents latency in first TTE network, whereas the green line represents latency in second TTE network.

We don't focus on RC and BE traffic in the network as it's given the least priority as based on TT scheduling of the message. This information is also shown in a node histogram in FAs, as shown in Figure 3 and 4. TT messages when it is prioritised, and message sent into the network the clocks are internally synchronised when simulating a wired message in a TTE network. This shows how time is propagated with an internal clock, highlighting low latency in the network.

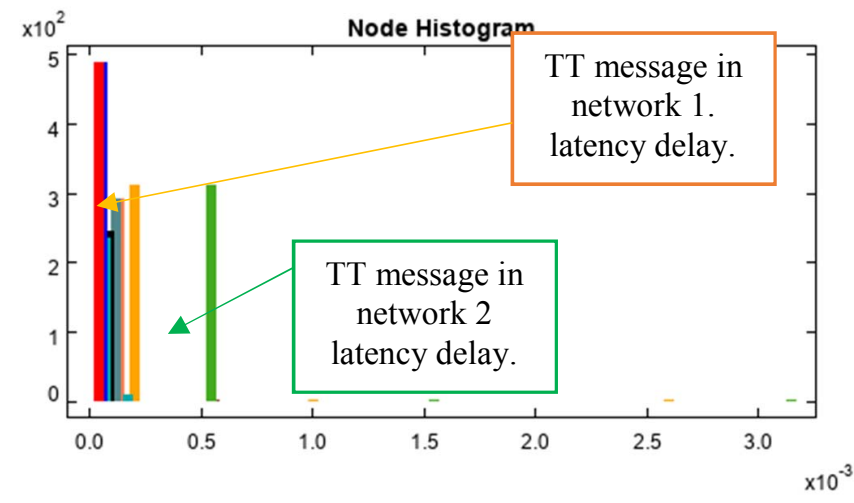

Fig. 4. Proposed scenario with multiple TTE networks in a wired connection.

In Figure 3 and 4 we see how internal clock synchronization shows low latency in the network as clocks are internally synchronized and the messages are sent within given time period. With the use of wireless communication link in a wired network we try to complement TTE technology with an external GNSS| clock as the main time source in the network. We have addressed this by modifying MAC layer of the OSI model to support for a wireless ES to be connected to the main TTE network this forms a SoS.

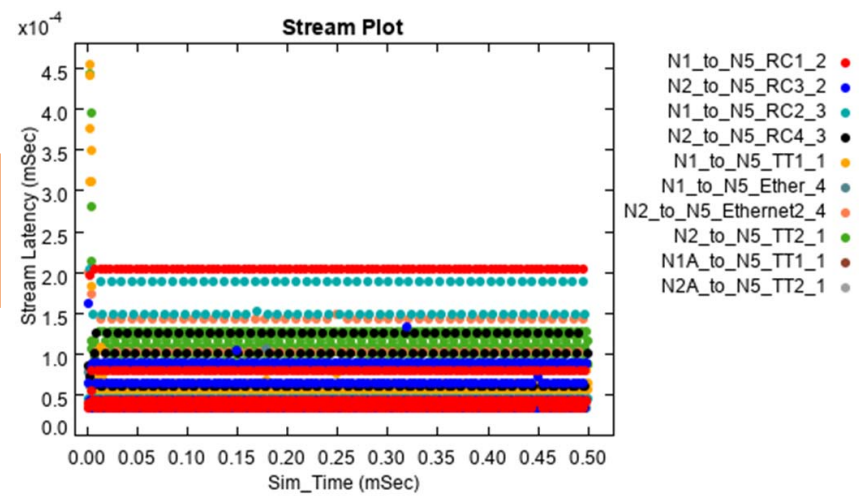

Fig. 1. Stream plot with 64 header bytes showing latency in the network.

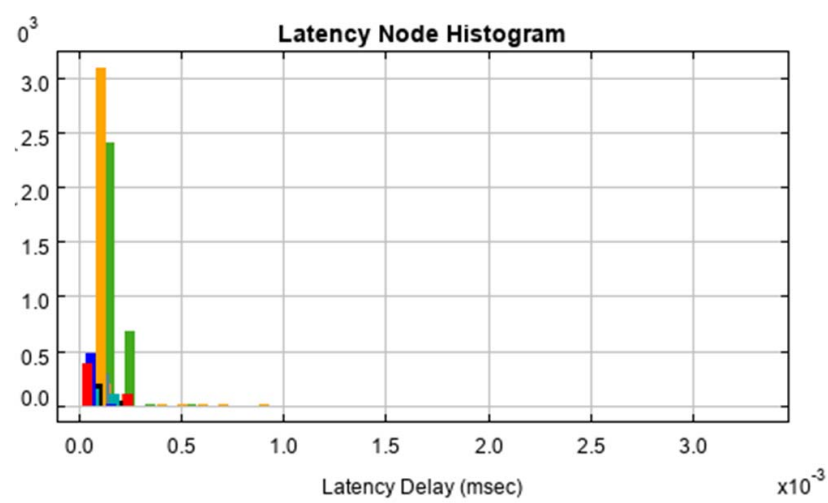

Fig. 2. Node histogram of latency in the TT network with 64 bytes and GNSS.

Figure 5 and 6 show how the QoS of the network gets effected as compared to a smaller header byte. The key point to 
notie here is that the green and yellow columns are the TT messages in the multiple networks with a quicker time to propagate the TT traffic in the network.

Figure 7 shows when connected with a GPS module latency increases and can be observed in TTE multiple network.

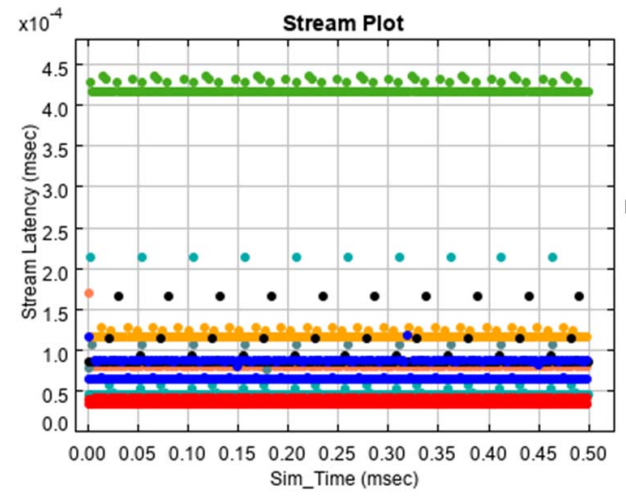

N1_to_N5_RC1_2 N2_to_N5_RC3_2 N1_to_N5_RC2_3 N2_to_N5_RC4_3. N1_to_N5_TT1_1 N1_to_N5_Ether_4 N2_to_N5_Ethernet2_4 N2_to_N5_TT2_1 N1A_to_N5_TT1_1 N2A_to_N5_TT2_1

Fig. 3. GPS connected ES in a TT network.

The above figure shows that latency increases significantly when a GNSS device is connected via USB to ES. This happens although we use the same header bytes 16 and 64 bytes for network 1 and network 2. As TT messages propagate in the network from ES1 to n...ES with a GNSS device connected to ES1, the latency in the network increases then compared to previous setup. The increase in latency is due to the fact that when a GNSS device is connected via USB, there is delay in communication between the USB and the ES. Then there is a cold start delay by the GPS receiver. These are the main reasons for increased latency compared to a non-GNSS connected device.

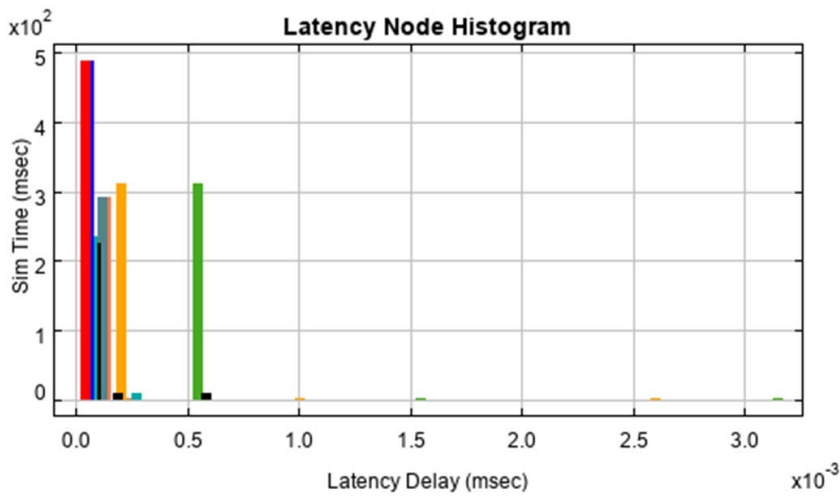

Fig. 4. Histogram plot GNSS connected latency in TT network.

The histogram in Figure 8 shows a significant delay in latency when the network is connected to an external time source. This occurs when we attached a wireless ES to an access point through two bridges to form a SoS in the network.

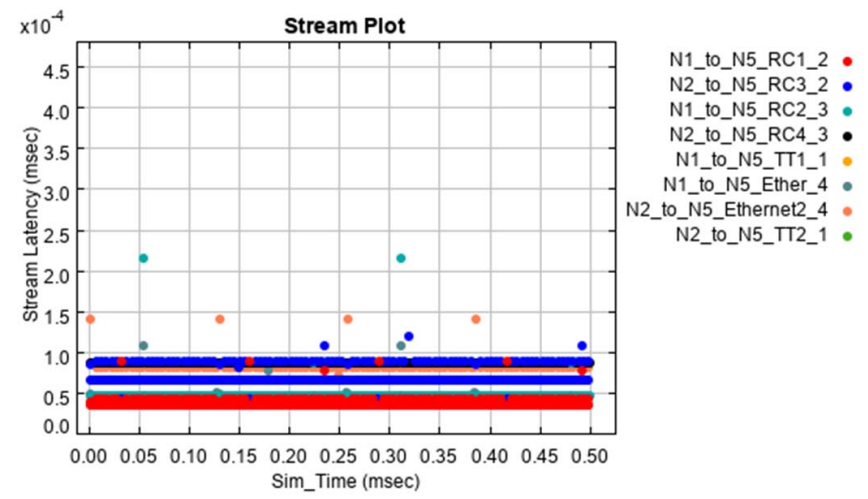

Fig. 5. Wirelessly connected ES with GPS as external clock.

Figure 9 illustrates the wireless connected ES with GNSS as external clock to prove how TT messages in the WES has less latency in the entire network. Hence, we have simulation results of one node connected as wireless whereas other ES are connected as wired connections and GNSS time is propagated through the network.

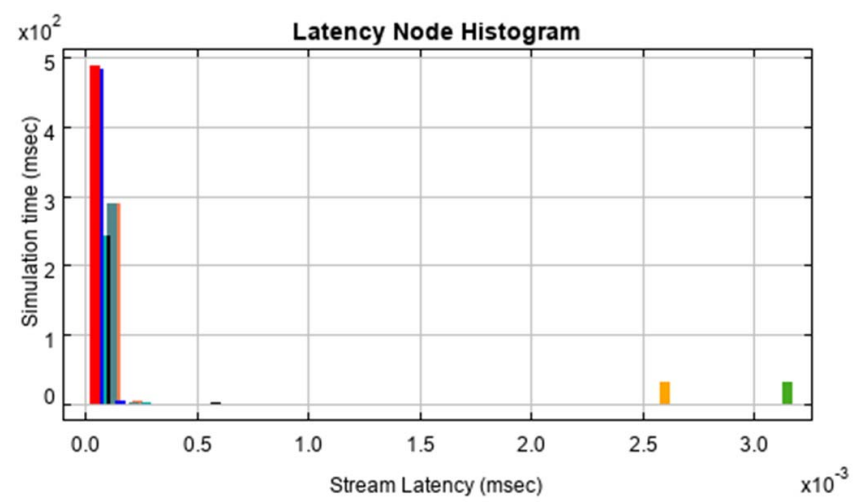

Fig. 6. Latency in a WES simulated network

Figure 10 shows a significant latency in the TT network when connecting a wireless ES through an access point compared to other network scenarios. It is important to point out here that a WES is connected to the network through an access point. This access point is also connected to the main TTE network through the TT bridge.

\section{SYSTEM ANALYSIS AND DISCUSSION}

In the simulation we had $4 \mathrm{ES}$ with two bridges acting as a redundant network and acts as a multiple TT network. In this we will evaluate the overall delay in the network for TT message we will ignore other traffic in the network that is $\mathrm{RC}$ and $\mathrm{BE}$ traffic. In a second experiment, we do a head-to-head comparison of GNSS connected TT network against a nonGNSS connected network. Our experiments reveal the following key findings: 
- When a GNSS device is connected to an ES on a TT network the latency increases as there is a delay due to USB stack of GNSS.

- One of the key benefits from enabling a GNSS time base in a TTE network is a support of multiple TT systems (aerial networks and TTE networks).

- The latency in the network increases when an external time source, such as a GNSS device, is introduced.

- The overall performance of a TTE network is degraded when a wireless ES is introduced in the network: latency is increased.

Table I represents the latency in TT network using 64-byte header as the message size the network uses its own PCF algorithm to propagate time in the network. The results highlight the minimum latency in the network is around 55 us whereas the maximum delay in the network is $2.693 \mathrm{~ms}$ whereas the throughput at first interval is $9.58 \mathrm{Mbps}$ and starts to decrease as the TT traffic is propagated in multiple networks and it switches from network one to network other (ES1 and ES1A).

TABLE I. LATENCY WITH INTERNAL CLOCK IN TT NETWORK

\begin{tabular}{|l|l|l|l|}
\hline \multirow{2}{*}{$\begin{array}{c}\text { Type of Traffic } \\
\text { TT Node 1 n }\end{array}$} & \multicolumn{3}{|c|}{$\begin{array}{c}\text { TT network with Internal clock } \\
\text { synchronization }\end{array}$} \\
\cline { 2 - 4 } & $\begin{array}{c}\text { Minimum } \\
\text { Latency }\end{array}$ & $\begin{array}{c}\text { Maximum } \\
\text { Latency }\end{array}$ & $\begin{array}{c}\text { Throughpu } \\
\text { t Mbps }\end{array}$ \\
\hline ES1_to_ES5_TT1_1 & $55.4616 \mathrm{us}$ & $\begin{array}{c}2.69338 \mathrm{~ms} \\
2.51546 \mathrm{~ms}\end{array}$ & 9.586644 \\
\hline ES2_to_ES5_TT2_1 & $55.46166 \mathrm{us}$ & $3.01546 \mathrm{~ms}$ & 9.573765 \\
\hline ES1A_to_ES5_TT1_1 & $55.46166 \mathrm{us}$ & $80.44334 \mathrm{us}$ & 5.645218 \\
\hline ES2A_to_ES5_TT2_1 & $55.46166 \mathrm{us}$ & $86.18166 \mathrm{us}$ & 3.279356 \\
\hline
\end{tabular}

TABLE II. LATENCY WITH GNSS AS EXTERNAL CLOCK IN TT NETWORK

\begin{tabular}{|l|l|l|l|}
\hline \multirow{2}{*}{$\begin{array}{c}\text { Type of Traffic } \\
\text { TT Node } 1 \text { to n }\end{array}$} & \multicolumn{3}{|c|}{$\begin{array}{c}\text { TT network with GNSS external clock } \\
\text { synchronization }\end{array}$} \\
\cline { 2 - 4 } & $\begin{array}{c}\text { Minimum } \\
\text { Latency }\end{array}$ & $\begin{array}{c}\text { Maximum } \\
\text { Latency }\end{array}$ & $\begin{array}{c}\text { Throughpu } \\
\text { t Mbps }\end{array}$ \\
\hline ES1_to_ES5_TT1_1 & $115.46166 \mathrm{us}$ & $2.69338 \mathrm{~ms}$ & 0.959778 \\
\hline ES2_to_ES5_TT2_1 & $415.46166 \mathrm{us}$ & $3.22202 \mathrm{~ms}$ & 0.959201 \\
\hline ES1A_to_ES5_TT1_1 & $115.46166 \mathrm{us}$ & $115.46166 \mathrm{us}$ & 3.303116 \\
\hline ES2A_to_ES5_TT2_1 & $415.46166 \mathrm{us}$ & $415.46166 \mathrm{us}$ & 1.697091 \\
\hline
\end{tabular}

Table II shows the latency results when a GNSS device is connected to ES1. The latency fluctuates as a result of changes in the QoS of surrounding wireless domain. The minimum latency in first network setup is around 115.46 us and increases to $2.69 \mathrm{~ms}$. Whereas in the second network ES1A the latency is same as first network due to that fact both networks were switched $\mathrm{ON}$ at the same time. This means that both networks were experiencing the same effects from wireless domain and impacts have changed at later stages.
TABLE III. LATENCY WITH ONE WIRELESS ES

\begin{tabular}{|c|c|c|c|}
\hline \multirow{2}{*}{$\begin{array}{c}\text { Type of Traffic } \\
\text { TT Node 1 to n }\end{array}$} & \multicolumn{3}{|c|}{$\begin{array}{c}\text { TT network with internal clock } \\
\text { synchronization }\end{array}$} \\
\cline { 2 - 4 } & $\begin{array}{c}\text { Minimum } \\
\text { Latency }\end{array}$ & $\begin{array}{c}\text { Maximum } \\
\text { Latency }\end{array}$ & $\begin{array}{c}\text { Throughput } \\
\text { Mbps }\end{array}$ \\
\hline ES1_to_ES5_TT1_1 & $2.516376 \mathrm{~ms}$ & $2.516376 \mathrm{~ms}$ & 0.098596 \\
\hline WES1_to_ES5_TT2_1 & $3.016376 \mathrm{~ms}$ & $3.016376 \mathrm{~ms}$ & 0.098497 \\
\hline
\end{tabular}

In Table III, we show the latency results when additional ES is connected wirelessly (this acts as a UAV) while the main ES is connected with GNSS via USB. As the clocks are synchronised, TT messages are propagated in the network. The results show latency increases dramatically between the wired and wireless ES in the network. The increase in latency is caused by the network when trying to synchronize to multiple time sources concurrently. Therefore, it is recommended to select one of those time sources as the main reference for the network while keeping the secondary time source on standby. Additionally, it is also possible to develop a switching mechanism to correlate both time sources and provide the most accurate one based on previous readings.

\section{CONCLUSIONS}

This paper presents the aerial network design and performance analysis using an external GNSS with timetriggered solution to facilitate the communication data link in the SoS. Simulation results were presented to demonstrate the superiority of the proposed system. Evaluations were conducted in order to assess the latency for a system, employing an external GNSS synchronization and internal to TTE networks time propagation. We have shown that employing external time source time requires adjustments to the network to prevent multiple time resources without having mechanism that correlates and selects the most accurate data. The results also highlight that when a wireless ES is connected to the TTE network with time base of a GNSS the QoS is degraded in the entire network. However, the aerial network connected with the TTE could be used to serve as main time source that can be synchronized with multiple networks forming a system of systems.

\section{REFERENCES}

[1]. FCC, "White Paper: The role of deployable aerial communications architecture in emergency communications and recommended next steps white paper," Sep 2011.

[2]. IEEE standard for a precision clock synchronization protocol for networked measurement and control systems. IEEE Std 1588-2008 (Revision of IEEE Std 1588-2002), pages c1-269, July 2008.

[3]. D. Gessner, J. Proenza, M. Barranco and A. Ballesteros, "A FaultTolerant Ethernet for Hard Real-Time Adaptive Systems," in IEEE Transactions on Industrial Informatics, vol. 15, no. 5, pp. 2980-2991, May 2019.

[4]. Z. Zheng, F. He, H. Li, and J. Lu, "Design Optimization of TimeTriggered Ethernet based on Routing and Scheduling Strategy," AIAA/IEEE Digit. Avion. Syst. Conf. - Proc., vol. 2019-Septe, 2019.

[5]. K. Bisson, "Sae As6802 Deterministic Ethernet Network Solution," Avion. Interface Technol., no. March, pp. 1-20, 2011, [Online]. Available: http://www.aviftech.com/. 
[6]. S. Li, Q. Ni, Y. Sun, G. Min and S. Al-Rubaye, "Energy-Efficient Resource Allocation for Industrial Cyber-Physical IoT Systems in 5G Era," in IEEE Transactions on Industrial Informatics, vol. 14, no. 6, pp. 2618-2628, June 2018.

[7]. P. G. Peón, H. Kopetz and W. Steiner, "Towards a reliable and high-speed wireless complement to TTEthernet," Proceedings of the 2014 IEEE Emerging Technology and Factory Automation (ETFA), Barcelona, 2014, pp. 1-4.

[8]. K. Steinhammer, P. Grillinger, A. Ademaj and H. Kopetz, "A TimeTriggered Ethernet (TTE) Switch," Proceedings of the Design Automation \& Test in Europe Conference, Munich, 2006, pp. 1-6.

[9]. P. G. Peón, E. Uhlemann, W. Steiner and M. Björkman, "Medium access control for wireless networks with diverse time and safety real-time requirements," IECON 2016 - 42nd Annual Conference of the IEEE Industrial Electronics Society, Florence, 2016, pp. 4665-4670.

[10]. C. Cicconetti, L. Lenzini, E. Mingozzi, and G. Stea, "Design and performance analysis of the Real-Time HCCA scheduler for IEEE 802.11e WLANs," Comput. Networks, 2007.

[11]. J. Son, H. Choi, and S. C. Park, "An effective polling MAC scheme for IEEE 802.11e," in IEEE International Symposium on Communications and Information Technologies, 2004.

[12]. H. Trsek and J. Jasperneite, "An isochronous medium access for real-time wireless communications in industrial automation systems - A use case for wireless clock synchronization," in IEEE International Symposium on Precision Clock Synchronization for Measurement, Control, and Communication, ISPCS, 2011.

[13]. A. Dongare, P. Lazik, N. Rajagopal and A. Rowe, "Pulsar: A Wireless Propagation-Aware Clock Synchronization Platform," 2017 IEEE RealTime and Embedded Technology and Applications Symposium (RTAS), Pittsburgh, PA, 2017, pp. 283-292.

[14]. S. Al-Rubaye and AntoniosTsourdos, "Airport Connectivity Optimization for 5G Ultra-Dense Networks," in IEEE Transactions on Cognitive Communications and Networking, early access, 2020.

[15].S. S. Craciunas, R. S. Oliver and V. Ecker, "Optimal static scheduling of real-time tasks on distributed time-triggered networked systems," Proceedings in the IEEE Emerging Technology and Factory Automation (ETFA), Barcelona, 2014. 
$2020-11-18$

\section{Quality of service study in synchronized time-triggered aerial networks}

Tariq, Nahman

IEEE

Tariq N, Petrunin I, Al-Rubaye S, et al., (2020) Quality of service study in synchronized time-triggered aerial networks. In: 2020 AIAA/IEEE 39th Digital Avionics Systems Conference (DASC), 11-15 October 2020,San Antonio, USA

https://doi.org/10.1109/DASC50938.2020.9256480

Downloaded from Cranfield Library Services E-Repository 